\title{
EFFICIENCY OF SOME INSECTICIDES SEQUENCE ON COTTON BOLLWORMS AND HISTOPATHOLOGICAL EFFECTS OF SOME BIOCIDES ON PINK BOLLWORM LARVAE
}

\author{
SALAMA, M. ABD EL-MOHSEN ${ }^{1}$, M. A. ABD EL-BAKI ${ }^{1}$, \\ JEHAN B.A. EL-NAGGAR ${ }^{2}$ and E.Y. EL-NAGAR ${ }^{2}$
}

1. Pesticides Dept., Fac. Agric., Kafrelsheikh Univ.

2. Plant Prot. Res. Inst., ARC, Egypt.

(Manuscript received 10 May 2012)

\begin{abstract}
The experiments were carried out during the cotton seasons of 2008 and 2009 in Sakha Agricultural Research Station Farm, Kafr El-Sheikh Governorate to evaluate the efficiency of certain insecticidal sequences against pink and spiny bollworms larvae. The aim of the insecticidal sequences is to get the most effective control one against the bollworms. The data revealed that the infestation of cotton fields with both bollworms in both successive seasons started with few number of $1^{\text {st }}$ and $2^{\text {nd }}$ instar larvae on late July, but increased gradually till the end of each season. In general all treatments had slight and moderate reduction in bollworms where the reduction rates ranged between 34.18 and $52.43 \%$. The sequences of biocides (Spinosad, Azadirachtin and Bacillus thuringiensis) was efficient in reducing larval population when sprayed three times alone or alternative with other three sprays at 15 days intervals. Spinosad in three successive sprays was the best sequences in controlling bollworms induced $48.66 \%$ average reduction, using the conventional insecticides with biocides, IGRs and antimolting compounds caused good average reduction on larval population. While the sequence of methomyl followed by oxymatrine + prosuler and lambdacyhalothrin induced the least reduction which being $38.02 \%$ reduction. With respect to the histopathological effects of the tested biocides, midgut histological sections were carried out on the $2^{\text {nd }}$ and $4^{\text {th }}$ instar larvae of Pink bollworm (PBW) with field concentration of the three tested biocides. Spinosad was the most effective compounds in causing aberrations in the midgut layers. Also, the pathological effect of the tested biocides was more serious on the $2^{\text {nd }}$ instar larvae than the $4^{\text {th }}$ one. Accordingly, spinosad, IGRs, antimolting compounds and biocides could be included with conventional insecticides to get the highest reduction in bollworms population.
\end{abstract}

\section{INTRODUCTION}

Cotton bollworms, the pink bollworm (PBW), Pectinophora gossypiella (Saunders) and the spiny bollworm (SPW), Earias insulana (Boisd.) are considered the most serious pests attacking cotton plants during flowering as well as fruiting stages and cause great losses of cotton yield and quality. Menally and Mullins (1996) indicated that the loss amount caused by $P$. gossypiella to cotton arises to one million 
kentar annually in Egypt. As a result of continued and unregulated application of insecticides, insects began to develop high levels of resistance to insecticides. In response to this problem effort to control or suppress bollworm damage to growing cotton frequently involve using insecticides of different groups in rotation program which may be useful to delay the resistance problem. Khidr et. al. (1996), El-Sorady et. al. (1998), and Abd El-Mageed et. al. (2007) indicated that application of insecticides in sequential use induced higher reduction in larval number as compared with the lower reduction resulting from several applications with the same insecticide alone. Use of the microbial insecticides, derived from Bacillus thuringiensis are nontoxic to human (Corlett, 1961), plants, animals, fishes and for parasitoids and predators (Burges and Hussey, 1971), natural products of plant origin is a new trend that preserve the environment from contamination with harmful toxicant.

Azadirachtin exhibits extremely low acute mammalian toxicity but it is very effective as control agent for many insect groups (Champagne et al., 1989). Also, biotic compound played an important role in pest control, among these compounds spinosad, it is a soil dwelling bacterium called Saccharopolyspora spinosa. Spinosad posses less rich than most insecticides to mammals, birds, fish and beneficial insects. It was used for control of Lepidoptera insect (Temarak, 2007, Ghure et. al., 2008 and Gosalwad et. al., 2009).

The present work is an attempt to evaluate the effectiveness of sequence insecticides mixtures with insect growth regulators IGRs and some biocide against cotton bollworms, (pink and spiny bollworms) infesting cotton green bolls in the cotton fields. Also, to study the histopathological effects of some biocides against either $2^{\text {nd }}$ and $4^{\text {th }}$ instar larvae of PBW, P. gossypiella.

\section{MATERIALS AND METHODS}

\section{Insecticides}

All tested insecticides used in this study were in formulated forms:

a. Organophosphates

a.1. Chlorpyrifos (Dursban $48 \%$ EC). It was supplied by Dow Agro Sciences, applied at rate of $1 \mathrm{~L} / \mathrm{fed}$.

a.2. Profenofos (Curacron $72 \%$ EC). It was supplied by syngenta, applied at rate $750 \mathrm{~mL} /$ fed.

b. Synthetic pyrethroids

b.1. Deltamethrin (Decis $2.5 \%$ EC) it was supplied by Bayer crop Science, applied at rate $750 \mathrm{ml} / \mathrm{fed}$. 
b.2. Lambda-cyhalothrin (Karate $2-5 \%$ EC). It was supplied by Samtrade Co., at rate $750 \mathrm{ml} / \mathrm{fed}$.

c. Carbamate

c.1. Methomyl (Lannate 90 WP). It was supplied by Bayer Crop Science, applied at rate $300 \mathrm{gm} / \mathrm{fed}$.

d. Oxadizine

d.1. Indoxacarb: (Steward $15 \%$ EC). It was supplied by Du Pont Agriculture, applied at rate $26.25 \mathrm{ml} / 100 \mathrm{~L}$.

e. Insect growth regulators (IGRs):

e.1. Chlorfluazuron (Atabron $5 \%$ EC). It was supplied by Ishinara Sangyo, applied at rate $400 \mathrm{ml} / \mathrm{fed}$.

e.2. Hexaflumuron (Consult $10 \%$ EC). It was supplied by Dow Agro Science, Applied at rate $200 \mathrm{ml} / \mathrm{fed}$.

e.3. Oxymatrine $0.4 \%+$ prosuler $0.2 \%$ (Kingo $0.6 \%$ S.L). It was supplied by King Bo, China, applied at rate $200 \mathrm{ml} /$ fed.

f. Biocides

f.1. Bacillus thuringiensis (Agerin 6.4\% WP). It was supplied by Plant Genetic Engineering Research Institute, applied at rate $500 \mathrm{gm} / \mathrm{fed}$.

f.2. Spinosad (Tracer $24 \%$ SC). It was supplied by Dow Agro Sciences, applied at rate $50 \mathrm{ml} / \mathrm{fed}$.

f.3. Azadirachtin (Neem-Azal $0.15 \%$ EC). It was supplied by cyclo-forune Grtis Tagros, applied at rate $600 \mathrm{ml} / \mathrm{fed}$.

\section{Design of field experiments}

The effect of different insecticides in 19 rotations against the pink bollworm and spiny bollworm (SBW), E. insulana (Boisd.) infesting cotton plants was studied to select the best sequence. The experiment was carried out at Sakha Agricultural Research Station, Kafr El-Sheikh Governorate during 2008 and 2009 growing cotton seasons. The cultivated cotton variety was Giza 86 . The area was arranged in a complete randomized block design with four replicates for each treatment. Each replicate was one kirate (1/24 feddan). Tested compounds were sprayed using a knapsack sprayer (CP3) at rate of 200 Liter/feddan. All treatments were done when the percent of bollworms infestation exceeded $3 \%$. Table (1) summarized the different sequential treatments and dates of spraying. The interval between any two successive applications was two weeks. 
Table 1. Represent different sequential treatments during 2008 and 2009 cotton season.

\begin{tabular}{|c|c|c|c|}
\hline \multirow[b]{2}{*}{ No. } & \multicolumn{3}{|c|}{ Sequence of treatment } \\
\hline & $\begin{array}{c}1^{\text {st }} \text { spray } \\
10 / 8 / 2008 \\
29 / 7 / 2009 \\
\end{array}$ & $\begin{array}{c}2^{\text {nd }} \text { spray } \\
24 / 8.2008 \\
11 / 8 / 2009 \\
\end{array}$ & $\begin{array}{c}3^{\text {rd }} \text { spray } \\
7 / 9 / 2008 \\
25 / 8 / 2009\end{array}$ \\
\hline 1 & $\begin{array}{c}\text { King Bo. } \\
\text { (Oxymatrine+ prosaler) }\end{array}$ & Lambdacyhalothrin & Methomyl \\
\hline 2 & Indoxacarb & Chlorpyrifos & Deltamethrin \\
\hline 3 & Chlorfluazuron & Methomyl & Deltamethrin \\
\hline 4 & Hexaflumuron & Deltamethrin & Profenofos \\
\hline 5 & Chlorpyrifos & Deltamethrin & $\begin{array}{c}\text { King Bo. } \\
\text { (Oxymatrine+ prosaler) }\end{array}$ \\
\hline 6 & Methomyl & $\begin{array}{c}\text { King Bo. } \\
\text { (Oxymatrine+ prosaler) }\end{array}$ & Lambda cyhalothrin \\
\hline 7 & Profenfos & Lambda cyhalothrin & Methomyl \\
\hline 8 & Spinosad & Spinosad & Spinosad \\
\hline 9 & B. thuringiensis (Agerin) & B. thuringiensis (Agerin) & B. thuringiensis (Agerin) \\
\hline 10 & Azadirachtin (Neem) & Azadirachtin (Neem) & Azadirachtin (Neem) \\
\hline 11 & Spinosad & B. thuringiensis (Agerin) & Azadirachtin (Neem) \\
\hline 12 & B. thuringiensis (Agerin) & Spinosad & Azadirachtin (Neem) \\
\hline 13 & Azadirachtin (Neem) & B. thuringiensis (Agerin) & Spinosad \\
\hline 14 & Spinosad & Lambda cyhalothrin & Methomyl \\
\hline 15 & Indoxacarb & Spinosad & Lambdacyhalothrin \\
\hline 16 & Chlorfluazuron & B. thuringiensis (Agerin) & Chlorpyrifos \\
\hline 17 & Hexaflumuron & Azadirachtin (Neem) & Deltamethrin \\
\hline 18 & B. thuringiensis (Agerin) & Deltamethrin & $\begin{array}{c}\text { King Bo. } \\
\text { (Oxymatrine+ prosaler) }\end{array}$ \\
\hline 19 & Azadirachtin (Neem) & King bo & Lambdacyhalothrin \\
\hline 20 & Untreated & & \\
\hline
\end{tabular}

\section{2.a. Determination of bollworms infestation}

For assessing the infestation by the pink and spiny bollworms, 100 green bolls were collected weekly at random from both diagonals of the inner square area for each plot ( 25 bolls for each replicate) according to the method of Shaaban and Radwan (1974). 


\section{Laboratory experiment}

a. Rearing of pink bollworm

Newly hatched larvae of $P$. gossypiella were obtained from the Bollworms Research Department, Plant Protection Research Institute, Dokki, Giza, Egypt and reared several generations on modified artificial diet at $27 \pm 1^{\circ} \mathrm{C}$ and $75 \pm 5 \%$ R.H. as described by Abd El-Hafez et. al. (1982).

\section{b. Histopathological studies}

Detection of chronic effects of the tested biocides against either $2^{\text {nd }}$ or $4^{\text {th }}$ instar larvae of pink bollworm treated at newly hatched larvae was as follows:

The newly hatched larvae of PBW were fed on diets containing field recommended concentrations of the tested biocides. These rates were $0.25 \mathrm{mg} / \mathrm{L}, 3.0$ $\mathrm{ml} / \mathrm{L}, 3 \mathrm{~g} / \mathrm{L}$ for spinosad, Azadirachtin and Agrin, respectively, then held at $27 \pm 1^{\circ} \mathrm{C}$ and $75 \pm 5$ R.H. for two days after treatment. The survivors $2^{\text {nd }}$ and $4^{\text {th }}$ larvae were transferred to glass vials $(2 \times 7 \mathrm{~cm})$ containing untreated diet, the survived $2^{\text {nd }}$ and $4^{\text {th }}$ instar larvae ( 7 and 14 days old) were removed and fixed in formalin $10 \%$ and processed to paraffin sections according to the method of Lee (1950). The same procedure was adopted on untreated larvae as comparison. All histopathological study was done in the Dept. of Zoology, Faculty of Science, Cairo University.

\section{Statistical analysis}

The reduction percentages of bollworms infestation were calculated according to the equation of Henderson and Tilton (1955). All data were subjected to one-way analysis of variance (ANOVA) followed by Duncan multiple range test (Duncan, 1955) to determine the significant differences among treatments mean values at 0.05 probability .

\section{RESULTS AND DISCUSSION}

The effectiveness of the insecticidal sequence for the reduction in the green boll infestation caused by pink and spiny bollworms were summarized and discussed as follows:

Data presented in Table (2) showed that the numbers of bollworms larvae per 25 green bolls during 2008 cotton season. It is clear that the infestation with both pink and spiny bollworms started with few numbers of $1^{\text {st }}$ and $2^{\text {nd }}$ instar larvae from 
late July and slightly increased gradually till the end of season. Also, all insecticides treatments decreased significantly the population of both pests compared to the untreated check as the mean number of bollworms. Larvae/25 bolls in treated plots ranged between 1.42 and 4.08 larvae, whereas it reached to 10.29 larvae in untreated plots.

Comparing between the efficiency of the tested insecticidal sequences against both bollworms, the data presented in Table (3) revealed that spraying of Hexafluzuron followed by methomyl and profenofos (sequence 4) was the most effective insecticidal treatment induced $50.05 \%$ reduction in larval population, while the sequence number (16) included chlorfluzuron, B. thuringiensis and chlorpyrifos was the least effective sequence with percent reduction of $34.18 \%$ comparing between the different insecticidal sequences against bollworms infestation, Based on general mean reduction in bollworms infestation the potency of these sequences could be arranged descendingly as the following sequence number: $4(50.05 \%, 13$ (46.99\%), 8 (44.88\%), 17 (44.72\%), $11(43.78 \%), 1(43.49 \%)$, S7 (43.22\%), 10 $(43.10 \%), 12(41.06 \%), 3(40.27 \%), 18(40.07 \%), 5(39.67 \%), 9(38.92 \%), 6$ (37.38\%), 15 (37.12\%), 19 (37.12\%), 2 (37.04\%), 14 (36.09\%) and $16(34.18 \%)$ reduction in larval population. With regard to 2009 cotton season, data presented in Table (4) showed similar trend of results as in 2008 season, but the effect of tested insecticidal sequences against both bollworms were relatively more than in the first season, the mean number of bollworms larvae per 25 boll in treated plots ranged between 1.79 and 3.71 larvae, while it reached to 10.25 larvae in the untreated plots. Data presented in Table (5) indicated that repetitive spraying of spinosad (biocide) in the three spraying (sequence 8) was the most efficient insecticidal treatment induced $52.43 \%$ reduction in larval population compound with other sequences, on contrary the sequence number (6) was the least effective sequence with percent reduction of $38.65 \%$. Based an general mean reduction in boll worms infestation the potency of these sequences could be arranged, discerningly as the following sequence number: 8 (52.43\%), 11 (47.76\%), 18 (46.83\%), 16 (46.16\%), 17 (45.83\%), $12(45.82 \%), 7$ (43.94), 1 (43.82\%), 13 (43.78\%), 9 (43.58\%), 14 (43.17\%), 2 (42.77\%), 19 (42.74\%), $15(42.63 \%), 10(41.99 \%), 3(40.76 \%), 5(39.86 \%), 4(39.09 \%)$ and $6(38.65 \%)$ reduction in larval population (Table 5). 
Table 2. Effect of different sequence of insecticides on the number of bollworms larvae/25 cotton green bolls during 2008 cotton season

\begin{tabular}{|c|c|c|c|c|c|c|c|c|c|c|c|c|c|c|c|c|}
\hline \multirow{2}{*}{$\begin{array}{l}\text { No. of } \\
\text { seq. }\end{array}$} & \multicolumn{3}{|c|}{ Sequences } & \multicolumn{4}{|c|}{\begin{tabular}{|c|} 
After $1^{\text {st }}$ spray \\
Week
\end{tabular}} & \multicolumn{4}{|c|}{ After $2^{\text {na }}$ spray } & \multicolumn{4}{|c|}{ After $3^{\text {to }}$ spray } & \multirow{2}{*}{$\begin{array}{c}\text { Genera } \\
\text { mean }\end{array}$} \\
\hline & $1^{s t}$ & $2^{\text {na }}$ & $3^{\text {ra }}$ & \begin{tabular}{|l|} 
Before \\
spray
\end{tabular} & $1 \mathrm{w}$ & $\frac{\sqrt{\text { leek }}}{2 w}$ & $\begin{array}{l}\text { ter } \\
\text { Mean }\end{array}$ & $\begin{array}{l}\text { Before } \\
\text { spray }\end{array}$ & $1 \mathrm{w}$ & $\begin{array}{l}\text { Week } \\
2 \mathrm{w}\end{array}$ & $\frac{1 \text { ter }}{\text { Mean }}$ & $\begin{array}{l}\text { Before } \\
\text { spray }\end{array}$ & $1 \mathrm{w}$ & $\frac{\text { Week a }}{2 \mathrm{w}}$ & Mean & \\
\hline 1 & Oxymatrine + Prosuler & Lambdacyhalothrin & Methomyl & 1.50 & 2.00 & 2.75 & $1.19 \mathrm{bcd}$ & 2.75 & 3.50 & 3.25 & $3.37 \mathrm{de}$ & 3.25 & 2.25 & 3.00 & $2.63 \mathrm{efg}$ & $2.79 \mathrm{~b}$ \\
\hline 2 & Indoxacarb & Chlorpyrifos & Deltamethrin & 1.25 & 1.75 & 2.25 & $2.00 \mathrm{~b}-\mathrm{e}$ & 2.25 & 2.75 & 3.75 & 3.25 def & 3.75 & 3.50 & 3.50 & 3.26 def & $2.92 \mathrm{~b}$ \\
\hline 3 & Chlorfluazuron & Methomyl & Deltamethrin & 1.50 & 2.50 & 2.50 & $2.50 \mathrm{bc}$ & 2.50 & 2.75 & 3.00 & $2.87 \mathrm{e}-\mathrm{h}$ & 3.00 & 2.50 & 2.75 & $2.63 \mathrm{efg}$ & $2.66 \mathrm{~b}$ \\
\hline 4 & Hexaflumuron & Deltamethrin & Profenofos & 1.50 & 1.50 & 2.25 & 1.87 cde & 2.25 & 1.25 & 3.00 & $2.13 \mathrm{hi}$ & 3.00 & 2.50 & 2.75 & $2.63 \mathrm{efg}$ & $2.21 \mathrm{~b}$ \\
\hline 5 & Chlorpyrifos & Deltamethrin & Oxymatrine + Prosuler & 1.00 & 1.50 & 1.75 & $1.63 \mathrm{def}$ & 1.75 & 2.50 & 2.75 & $2.63 \mathrm{e}-\mathrm{h}$ & 2.75 & 2.25 & 2.50 & $2.37 \mathrm{fgh}$ & $2.25 \mathrm{~b}$ \\
\hline 6 & Methomyl & Oxymatrine + Prosuler & Lambdacyhalothrin & 1.00 & 1.50 & 2.0 & $1.75 c-f$ & 2.00 & 1.75 & 3.00 & $2.37 \mathrm{f}-\mathrm{i}$ & 3.00 & 3.50 & 3.50 & 3.50 def & $2.54 \mathrm{~b}$ \\
\hline 7 & Profenofos & Lambdacyhalothrin & Methomyl & 1.00 & 1.50 & 1.75 & $1.63 \mathrm{def}$ & 1.75 & 1.50 & 2.50 & $2.0 \mathrm{hi}$ & 2.50 & 2.00 & 2.50 & $2.25 \mathrm{gh}$ & $1.95 \mathrm{~b}$ \\
\hline 8 & Spinosad & Spinosad & Spinosad & 1.25 & 0.75 & 1.25 & $1.00 \mathrm{f}$ & 1.25 & 1.50 & 1.50 & $1.5 \mathrm{i}$ & 1.50 & 1.50 & 1.75 & $1.63 \mathrm{~h}$ & $1.38 \mathrm{~b}$ \\
\hline 9 & B. thuringiensis & B. thuringiensis & B. thuringiensis & 1.50 & 2.50 & 3.00 & $2.75 b$ & 3.00 & 3.50 & 4.00 & $3.75 \mathrm{~cd}$ & 4.00 & 3.75 & 3.25 & 3.5 cde & $3.33 b$ \\
\hline 10 & Azadirachtin & Azadirachtin & Azadirachtin & 1.25 & 1.25 & 2.75 & $2.00 \mathrm{~b}-\mathrm{e}$ & 2.75 & 4.00 & 5.00 & $4.5 \mathrm{bc}$ & 5.00 & 3.00 & 3.50 & 3.25 def & $3.25 b$ \\
\hline 11 & Spinosad & B. thuringiensis & Azadirachtin & 1.00 & 1.00 & 2.00 & 1.50 ef & 2.00 & 1.25 & 2.75 & $2.00 \mathrm{hi}$ & 2.75 & 2.50 & 3.25 & $2.87 \mathrm{~d}-\mathrm{g}$ & $2.13 \mathrm{~b}$ \\
\hline 12 & B. thuringiensis & Spinosad & Azadirachtin & 1.25 & 1.50 & 2.50 & $2.00 \mathrm{~b}-\mathrm{e}$ & 2.50 & 2.50 & 2.75 & $2.62 \mathrm{e}-\mathrm{h}$ & 2.75 & 2.50 & 3.25 & $2.87 \mathrm{~d}-\mathrm{g}$ & $2.50 \mathrm{~b}$ \\
\hline 13 & Azadirachtin & B. thuringiensis & Spinosad & 1.50 & 2.00 & 3.50 & $2.75 \mathrm{~b}$ & 3.50 & 3.50 & 5.25 & $4.37 \mathrm{bc}$ & 5.25 & 2.75 & 3.25 & $3.00 \mathrm{~d}-\mathrm{g}$ & $3.38 \mathrm{~b}$ \\
\hline 14 & Spinosad & Lambdacyhalothrin & Methomyl & 1.00 & 1.50 & 1.75 & $1.60 \mathrm{def}$ & 1.75 & 1.75 & 2.75 & 2.25 ghi & 2.75 & 2.75 & 3.25 & $3.00 \mathrm{~d}-\mathrm{g}$ & $2.29 \mathrm{~b}$ \\
\hline 15 & Indoxacarb & Spinosad & Lambdacyhalothrin & 1.25 & 1.50 & 2.00 & $1.75 \mathrm{c}-\mathrm{f}$ & 2.00 & 3.00 & 3.25 & $3.12 \mathrm{~d}-\mathrm{g}$ & 3.25 & 2.25 & 3.75 & 3.50 def & $2.63 \mathrm{~b}$ \\
\hline 16 & Chlorfluazuron & B. thuringiensis & Chlorpyrifos & 1.50 & 2.50 & 3.00 & $2.75 b$ & 3.00 & 4.50 & 5.00 & $4.75 \mathrm{~b}$ & 5.00 & 4.75 & 3.50 & $4.13 \mathrm{c}$ & $3.88 \mathrm{~b}$ \\
\hline 17 & Hexaflumuron & Azadirachtin & Deltamethrin & 1.50 & 1.00 & 3.00 & $2.00 \mathrm{~b}-\mathrm{e}$ & 3.00 & 3.50 & 3.25 & $3.37 \mathrm{de}$ & 3.25 & 3.00 & 3.00 & $3.00 \mathrm{~d}-\mathrm{g}$ & $2.79 \mathrm{~b}$ \\
\hline 18 & B. thuringiensis & Deltamethrin & Oxymatrine + Prosuler & 1.25 & 1.25 & 3.00 & $2.12 \mathrm{~b}-\mathrm{e}$ & 3.00 & 3.50 & 4.50 & $3.25 \mathrm{bcd}$ & 4.50 & 4.00 & 3.50 & $3.75 \mathrm{~cd}$ & $3.29 b$ \\
\hline 19 & Azadirachtin & Oxymatrine + Prosuler & Lambdacyhalothrin & 1.50 & 1.00 & 3.50 & $2.50 \mathrm{~b}-\mathrm{e}$ & 3.50 & 5.00 & 5.00 & $4.25 b$ & 5.00 & 5.50 & 4.50 & $5.00 \mathrm{~b}$ & $4.08 \mathrm{~b}$ \\
\hline 20 & Control & & & 1.50 & 5.00 & 5.00 & $5.00 \mathrm{a}$ & 5.00 & 8.25 & 11.5 & $6.62 \mathrm{a}$ & 11.50 & 14.50 & 17.50 & $16.00 \mathrm{a}$ & $10.29 \mathrm{a}$ \\
\hline
\end{tabular}

Mean followed by the same letter are not significantly different at 0.05 level of probability according to DMRT 
Table 3. Reduction percentages in bollworms applied with different insecticidal sequences during cotton season of 2008

\begin{tabular}{|c|c|c|c|c|c|c|c|c|c|c|c|c|c|}
\hline \multirow{3}{*}{$\begin{array}{l}\text { No. of } \\
\text { seq. }\end{array}$} & \multicolumn{3}{|c|}{ No. of sequence } & \multirow{2}{*}{\multicolumn{3}{|c|}{$\begin{array}{c}1^{\text {st }} \text { spray } \\
\% \text { red after }\end{array}$}} & \multirow{2}{*}{\multicolumn{3}{|c|}{$\begin{array}{c}2^{\text {nd }} \text { spray } \\
\% \text { red after }\end{array}$}} & \multirow{2}{*}{\multicolumn{3}{|c|}{$\begin{array}{c}3^{\text {rd }} \text { spray } \\
\% \text { red after }\end{array}$}} & \multirow{3}{*}{$\begin{array}{c}\text { General } \\
\text { mean of } \\
\text { reduction }\end{array}$} \\
\hline & \multirow{2}{*}{$1^{\text {st }}$} & \multirow{2}{*}{$2^{\text {nd }}$} & \multirow{2}{*}{$3 r d$} & & & & & & & & & & \\
\hline & & & & $1 \mathrm{w}$ & $2 w$ & Mean & $1 \mathrm{w}$ & $2 w$ & Mean & $1 \mathrm{w}$ & $2 w$ & Mean & \\
\hline 1 & Oxymatrine + Prosuler & Lambdacyhalothrin & Methomyl & 60.00 & 45.00 & $52.50 \mathrm{bc}$ & 22.78 & 48.62 & 35.7 a-d & 45.08 & 39.35 & $42.23 \mathrm{~b}$ & $43.49 \mathrm{~g}$ \\
\hline 2 & Indoxacarb & Chlorpyrifos & Deltamethrin & 57.92 & 46.00 & $51.96 \mathrm{bc}$ & 25.92 & 27.55 & 26.73 cde & 25.98 & 38.84 & $32.21 \mathrm{bcd}$ & 37.04 bc \\
\hline 3 & Chlorfluazuron & Methomyl & Deltamethrin & 50.00 & 50.00 & $50.00 \mathrm{bc}$ & 33.35 & 47.83 & $40.59 \mathrm{abc}$ & 20.68 & 39.75 & $30.21 \mathrm{bcd}$ & $40.27 \mathrm{e}$ \\
\hline 4 & Hexaflumuron & Deltamethrin & Profenofos & 70.00 & 55.00 & $62.50 \mathrm{ab}$ & 59.59 & 42.04 & $50.81 \mathrm{a}$ & 33.91 & 39.76 & $36.84 \mathrm{bc}$ & $50.05 \mathrm{i}$ \\
\hline 5 & Chlorpyrifos & Deltamethrin & Oxymatrine + Prosuler & 55.00 & 62.50 & $58.75 \mathrm{bc}$ & 30.99 & 31.69 & 31.34 b-e & 35.11 & 40.27 & $37.69 \mathrm{~b}$ & $39.67 \mathrm{de}$ \\
\hline 6 & Methomyl & Oxymatrine + Prosuler & Lambdacyhalothrin & 55.00 & 57.14 & 56.07 bc & 46.97 & 34.78 & $40.87 \mathrm{abc}$ & 7.47 & 23.33 & $15.40 \mathrm{e}$ & $37.38 \mathrm{bcd}$ \\
\hline 7 & Profenofos & Lambdacyhalothrin & Methomyl & 55.00 & 47.50 & $51.25 \mathrm{bc}$ & 48.24 & 31.69 & 39.97 a-d & 27.91 & 34.29 & $31.10 \mathrm{bcd}$ & $43.22 \mathrm{fg}$ \\
\hline 8 & Spinosad & Spinosad & Spinosad & 80.00 & 70.00 & $75.00 \mathrm{a}$ & 27.25 & 47.84 & 37.37 a-d & 20.87 & 23.33 & $22.10 \mathrm{de}$ & $44.88 \mathrm{gh}$ \\
\hline 9 & B. thuringiensis & B. thuringiensis & B. thuringiensis & 50.00 & 40.00 & $45.00 \mathrm{c}$ & 29.29 & 42.02 & 35.65 a-d & 25.63 & 46.60 & $36.12 \mathrm{bc}$ & 38.92 cde \\
\hline 10 & Azadirachtin & Azadirachtin & Azadirachtin & 70.00 & 34.00 & $52.00 \mathrm{bc}$ & 11.85 & 36.77 & $24.31 \mathrm{de}$ & 52.00 & 54.00 & $53.00 \mathrm{a}$ & $43.10 \mathrm{fg}$ \\
\hline 11 & Spinosad & B. thuringiensis & Azadirachtin & 70.00 & 40.00 & $55.00 \mathrm{bc}$ & 62.12 & 40.21 & $51.16 a$ & 27.90 & 22.33 & $25.12 \mathrm{cde}$ & $43.78 \mathrm{~g}$ \\
\hline 12 & B. thuringiensis & Spinosad & Azadirachtin & 64.00 & 40.00 & $52.00 \mathrm{bc}$ & 39.39 & 52.77 & $46.08 \mathrm{ab}$ & 27.91 & 22.33 & $21.12 \mathrm{cde}$ & 41.06 ef \\
\hline 13 & Azadirachtin & B. thuringiensis & Spinosad & 60.00 & 30.00 & $45.00 \mathrm{c}$ & 39.39 & 34.78 & $37.08 \mathrm{a}-\mathrm{d}$ & 58.45 & 59.32 & $58.93 a$ & $46.99 \mathrm{hi}$ \\
\hline 14 & Spinosad & Lambdacyhalothrin & Methomyl & 55.00 & 47.50 & $51.00 \mathrm{bc}$ & 39.39 & 31.69 & $35.54 \mathrm{a}-\mathrm{d}$ & 20.68 & 22.33 & $21.50 \mathrm{de}$ & $36.09 a b$ \\
\hline 15 & Indoxacarb & Spinosad & Lambdacyhalothrin & 64.00 & 52.00 & $58.00 \mathrm{bc}$ & 9.10 & 29.34 & $19.22 \mathrm{e}$ & 45.08 & 23.22 & $34.15 \mathrm{bc}$ & 37.12 bc \\
\hline 16 & Chlorfluazuron & B. thuringiensis & Chlorpyrifos & 50.00 & 40.00 & $45.00 \mathrm{c}$ & 9.10 & 27.53 & $18.331 \mathrm{e}$ & 24.64 & 54.00 & $39.32 \mathrm{~b}$ & $34.18 \mathrm{a}$ \\
\hline 17 & Hexaflumuron & Azadirachtin & Deltamethrin & 80.00 & 40.00 & $60.00 \mathrm{bc}$ & 29.29 & 52.90 & $41.90 \mathrm{abc}$ & 26.80 & 39.35 & $33.07 \mathrm{bcd}$ & $44.72 \mathrm{gh}$ \\
\hline 18 & B. thuringiensis & Deltamethrin & Oxymatrine + Prosuler & 70.00 & 28.00 & $49.00 \mathrm{bc}$ & 29.29 & 34.78 & $32.03 \mathrm{~b}-\mathrm{e}$ & 29.50 & 48.89 & $39.19 \mathrm{~b}$ & 40.07 e \\
\hline 19 & Azadirachtin & Oxymatrine + Prosuler & Lambdacyhalothrin & 80.00 & 30.00 & $55.00 \mathrm{bc}$ & 13.43 & 37.89 & $25.66 \mathrm{cde}$ & 20.68 & 40.85 & $30.76 \mathrm{bcd}$ & 37.12 bc \\
\hline
\end{tabular}

Mean followed by the same letter are not significantly different at 0.05 level of probability according to DMRT 
Table 4. Effect of different sequences of insecticides on the number of bollworms larvae/25 cotton green bolls during 2009 cotton season.

\begin{tabular}{|c|c|c|c|c|c|c|c|c|c|c|c|c|c|c|c|c|}
\hline \multirow{3}{*}{$\begin{array}{l}\text { No. of } \\
\text { seq. }\end{array}$} & \multirow{2}{*}{\multicolumn{3}{|c|}{ Sequences }} & \multicolumn{4}{|c|}{ After $1^{\text {st }}$ spray } & \multicolumn{4}{|c|}{ After $2^{\text {nd }}$ spray } & \multicolumn{4}{|c|}{ After $3^{\text {rd }}$ spray } & \multirow{3}{*}{$\begin{array}{c}\text { General } \\
\text { mean }\end{array}$} \\
\hline & & & & \multirow{2}{*}{\begin{tabular}{|c|} 
Before \\
spray
\end{tabular}} & \multicolumn{3}{|c|}{ Week after } & \multirow{2}{*}{$\begin{array}{l}\text { Before } \\
\text { spray }\end{array}$} & \multicolumn{3}{|c|}{ Week after } & \multirow{2}{*}{\begin{tabular}{|l|} 
Before \\
spray
\end{tabular}} & \multicolumn{3}{|c|}{ Week after } & \\
\hline & $1^{\text {st }}$ & $2^{\text {na }}$ & $3^{\text {ra }}$ & & $1 \mathrm{w}$ & $2 \mathrm{w}$ & Mean & & $1 \mathrm{w}$ & $2 w$ & Mean & & $1 \mathrm{w}$ & $2 \mathrm{w}$ & Mean & \\
\hline 1 & $\begin{array}{l}\text { Oxymatrine } \\
\text { Prosuler }\end{array}$ & ${ }^{+}$Lambdacyhalothrin & Methomyl & 1.00 & 1.50 & 1.50 & 1.50 cde & 1.50 & 2.00 & 3.50 & $2.75 \mathrm{f}-\mathrm{i}$ & 3.50 & 2.50 & 2.25 & $2.37 \mathrm{fg}$ & $2.21 \mathrm{~b}$ \\
\hline 2 & Indoxacarb & Chlorpyrifos & Deltamethrin & 1.00 & 1.50 & 2.00 & $1.75 \mathrm{~cd}$ & 2.00 & 3.00 & 3.00 & 3.00 e-h & 3.00 & 2.25 & 2.75 & $2.50 \mathrm{fg}$ & $2.42 \mathrm{~b}$ \\
\hline 3 & Chlorfluazuron & Methomyl & Deltamethrin & 1.25 & 1.50 & 3.50 & $2.50 \mathrm{~b}$ & 3.50 & 4.00 & 4.50 & $4.25 \mathrm{bc}$ & 4.50 & 4.25 & 4.75 & $4.50 \mathrm{~b}$ & $3.79 \mathrm{~b}$ \\
\hline 4 & Hexaflumuron & Deltamethrin & Profenofos & 1.00 & 1.25 & 1.50 & 1.37 cde & 1.50 & 2.00 & 3.00 & $2.50 \mathrm{~g}-\mathrm{j}$ & 3.00 & 2.75 & 3.50 & $3.12 \mathrm{c}-\mathrm{g}$ & $2.33 \mathrm{~b}$ \\
\hline 5 & Chlorpyrifos & Deltamethrin & Oxymatrine + Prosuler & 1.00 & 1.25 & 2.00 & 1.63 cde & 2.00 & 2.50 & 3.50 & 3.00 e-h & 3.50 & 2.75 & 3.50 & $3.12 \mathrm{c}-\mathrm{g}$ & $2.58 \mathrm{~b}$ \\
\hline 6 & Methomyl & Oxymatrine + Prosuler & Lambdacyhalo & 1.00 & 1.50 & 1.75 & 1.63 cde & 1.75 & 2.50 & 3.50 & 3.00 e-h & 3.50 & 2.50 & 3.00 & $2.75 \mathrm{~d}-\mathrm{g}$ & $2.46 \mathrm{~b}$ \\
\hline 7 & Profenofos & Lambdacyhalothrin & Methomyl & 1.00 & 1.25 & 1.75 & 1.50 cde & 1.75 & 1.75 & 2.25 & $1.75 \mathrm{ij}$ & 2.25 & 2.25 & 2.75 & $2.50 \mathrm{fg}$ & $2.00 \mathrm{~b}$ \\
\hline 8 & Spinosad & Spinosad & Spinosad & 1.00 & 0.75 & 1.25 & $1.00 \mathrm{e}$ & 1.25 & 1.25 & 2.50 & $1.87 \mathrm{j}$ & 2.50 & 2.00 & 3.00 & $2.50 \mathrm{fg}$ & $1.79 \mathrm{~b}$ \\
\hline 9 & B. thuringiensis & B. thuringiensis & B. thuringiensis & 1.25 & 1.50 & 2.25 & $1.87 \mathrm{bc}$ & 2.25 & 2.50 & 4.50 & $3.50 c-j$ & 4.50 & 3.00 & 5.25 & $4.13 \mathrm{bc}$ & $3.17 \mathrm{~b}$ \\
\hline 10 & Azadirachtin & Azadirachtin & Azadirachtin & 1.00 & 0.75 & 1.75 & 1.25 cde & 1.75 & 2.00 & 3.00 & $2.50 \mathrm{~g}-\mathrm{j}$ & 3.00 & 3.25 & 3.50 & $3.37 \mathrm{c}-\mathrm{g}$ & $2.38 \mathrm{~b}$ \\
\hline 11 & Spinosad & B. thuringiensis & Azadirachtin & 0.75 & 0.75 & 1.50 & $1.13 \mathrm{de}$ & 1.50 & 2.00 & 2.75 & 3.37 hij & 2.75 & 2.00 & 2.50 & $2.25 \mathrm{~g}$ & $1.92 \mathrm{~b}$ \\
\hline 12 & B. thuringiensis & Spinosad & Azadirachtin & 1.25 & 0.75 & 2.25 & 1.50 cde & 2.25 & 2.50 & 3.00 & $2.75 \mathrm{f}-\mathrm{i}$ & 3.00 & 2.75 & 4.00 & $3.37 \mathrm{c}-\mathrm{g}$ & $2.51 \mathrm{~b}$ \\
\hline 13 & Azadirachtin & B. thuringiensis & Spinosad & 1.00 & 0.75 & 2.50 & 1.63 cde & 2.50 & 3.25 & 4.50 & $3.87 \mathrm{bcd}$ & 4.50 & 2.75 & 4.75 & $3.75 \mathrm{bcd}$ & $3.08 \mathrm{~b}$ \\
\hline 14 & Spinosad & Lambdacyhalothrin & Methomyl & 1.25 & 1.50 & 2.00 & $1.75 \mathrm{~cd}$ & 2.00 & 3.00 & 3.75 & 3.37 def & 3.75 & 2.25 & 3.00 & $2.63 \mathrm{efg}$ & $2.58 \mathrm{~b}$ \\
\hline 15 & Indoxacarb & Spinosad & Lambdacyhalot & 1.50 & 1.50 & 3.50 & $2.50 \mathrm{~b}$ & 3.50 & 3.75 & 5.00 & $4.37 \mathrm{~b}$ & 5.00 & 4.00 & 4.00 & $4.00 \mathrm{bc}$ & $3.63 \mathrm{~b}$ \\
\hline 16 & Chlorfluazuron & B. thuringiensis & Chlorpyrifos & 1.25 & 1.50 & 1.50 & 1.50 cde & 1.50 & 2.00 & 3.50 & $2.75 \mathrm{f}-\mathrm{i}$ & 3.50 & 2.75 & 2.50 & $2.63 \mathrm{efg}$ & $2.29 \mathrm{~b}$ \\
\hline 17 & Hexaflumuron & Azadirachtin & Deltamethrin & 1.25 & 1.00 & 2.50 & $1.75 \mathrm{~cd}$ & 2.50 & 2.50 & 4.00 & $3.25 \mathrm{~d}-\mathrm{g}$ & 4.00 & 2.75 & 4.50 & $3.63 \mathrm{~b}-\mathrm{e}$ & $2.88 \mathrm{~b}$ \\
\hline 18 & B. thuringiensis & Deltamethrin & Oxymatrine + Prosuler & 1.25 & 1.25 & 2.00 & 1.63 cde & 2.00 & 3.00 & 4.50 & 3.75 b-e & 4.50 & 4.00 & 5.00 & $4.54 \mathrm{~b}$ & $3.29 \mathrm{~b}$ \\
\hline 19 & Azadirachtin & Oxymatrine + Prosuler & Lamb & 1.25 & 1.50 & 3.00 & 2.25 cde & 3.00 & 4.00 & 4.00 & 4.00 b-e & 4.00 & 2.75 & 4.50 & $3.63 b-e$ & $.29 \mathrm{~b}$ \\
\hline 20 & Cont. & & & 1.00 & 3.25 & 4.50 & $3.87 \mathrm{a}$ & 4.50 & 8.50 & 11.75 & $10.00 \mathrm{a}$ & 11.75 & 14.50 & 19.00 & $16.75 \mathrm{a}$ & $10.25 a$ \\
\hline
\end{tabular}


Table 5. Reduction percentages in bollworms applied with different insecticidal sequences during cotton season of 2009

\begin{tabular}{|c|c|c|c|c|c|c|c|c|c|c|c|c|c|}
\hline \multirow{3}{*}{$\begin{array}{l}\text { No. of } \\
\text { seq. }\end{array}$} & \multicolumn{3}{|c|}{ No. of sequence } & \multirow{2}{*}{\multicolumn{3}{|c|}{$\begin{array}{c}1^{\text {st }} \text { spray } \\
\% \text { red after }\end{array}$}} & \multirow{2}{*}{\multicolumn{3}{|c|}{$\begin{array}{c}2^{\text {nd }} \text { spray } \\
\% \text { red after }\end{array}$}} & \multirow{2}{*}{\multicolumn{3}{|c|}{$\begin{array}{c}3^{\text {rd }} \text { spray } \\
\% \text { red after }\end{array}$}} & \multirow{3}{*}{$\begin{array}{l}\text { General mean of } \\
\text { reduction }\end{array}$} \\
\hline & \multirow{2}{*}{$1^{\text {st }}$} & \multirow{2}{*}{$2^{\text {nd }}$} & \multirow{2}{*}{$3 r d$} & & & & & & & & & & \\
\hline & & & & $1 \mathrm{w}$ & $2 w$ & Mean & $1 \mathrm{w}$ & $2 w$ & Mean & $1 \mathrm{w}$ & $2 w$ & Mean & \\
\hline 1 & Oxymatrine + Prosuler & Lambdacyhalothrin & Methomyl & 53.84 & 66.66 & 60.25 b-e & 29.41 & 10.66 & $20.04 \mathrm{e}$ & 42.12 & 60.24 & $51.18 \mathrm{a}$ & $43.82 \mathrm{e}$ \\
\hline 2 & Indoxacarb & Chlorpyrifos & Deltamethrin & 53.84 & 55.55 & $54.69 \mathrm{c}-\mathrm{f}$ & 20.00 & 44.68 & $32.34 \mathrm{~cd}$ & 39.22 & 43.32 & $41.27 \mathrm{abc}$ & $42.77 \mathrm{de}$ \\
\hline 3 & Chlorfluazuron & Methomyl & Deltamethrin & 63.10 & 37.50 & 50.30 ef & 39.49 & 50.76 & $44.75 a b$ & 18.96 & 34.72 & 26.84 def & $40.76 \mathrm{bc}$ \\
\hline 4 & Hexaflumuron & Deltamethrin & Profenofos & 61.53 & 66.66 & 64.09 a-d & 29.40 & 23.38 & 26.39 cde & 25.72 & 27.85 & 26.78 def & $39.09 \mathrm{a}$ \\
\hline 5 & Chlorpyrifos & Deltamethrin & Oxymatrine + Prosuler & 55.00 & 47.00 & 51.00 def & 30.99 & 31.69 & 31.34 cde & 36.33 & 38.16 & $37.25 \mathrm{bcd}$ & $39.86 a b$ \\
\hline 6 & Methomyl & Oxymatrine + Prosuler & Lambdacyhalothrin & 55.00 & 40.00 & $47.50 \mathrm{f}$ & 24.40 & 23.39 & $23.89 \mathrm{e}$ & 42.12 & 47.00 & $44.56 a b$ & $38.65 \mathrm{a}$ \\
\hline 7 & Profenofos & Lambdacyhalothrin & Methomyl & 61.38 & 61.11 & 61.24 a-e & 47.05 & 50.72 & $48.88 \mathrm{a}$ & 18.06 & 24.42 & $21.24 \mathrm{f}$ & $43.94 \mathrm{e}$ \\
\hline 8 & Spinosad & Spinosad & Spinosad & 76.92 & 72.22 & 74.57 a & 47.05 & 57.45 & $52.29 \mathrm{a}$ & 35.17 & 25.79 & $30.48 c-f$ & $52.43 \mathrm{~h}$ \\
\hline 9 & B. thuringiensis & B. thuringiensis & B. thuringiensis & 63.10 & 60.00 & 61.55 a-e & 41.19 & 23.41 & $32.30 \mathrm{~cd}$ & 45.98 & 27.85 & $36.92 \mathrm{bcd}$ & $43.58 \mathrm{de}$ \\
\hline 10 & Azadirachtin & Azadirachtin & Azadirachtin & 76.92 & 61.11 & $69.02 a b$ & 39.52 & 34.33 & $36.93 \mathrm{bc}$ & 12.21 & 27.85 & $20.03 \mathrm{f}$ & $41.99 \mathrm{~cd}$ \\
\hline 11 & Spinosad & B. thuringiensis & Azadirachtin & 76.92 & 66.66 & $71.79 a b$ & 29.41 & 29.78 & 29.59 cde & 41.07 & 43.78 & $42.42 a b c$ & $47.76 \mathrm{~g}$ \\
\hline 12 & B. thuringiensis & Spinosad & Azadirachtin & 81.52 & 60.00 & $70.76 a b$ & 41.19 & 48.94 & $45.06 \mathrm{ab}$ & 25.72 & 17.53 & $21.62 \mathrm{ef}$ & $45.82 \mathrm{f}$ \\
\hline 13 & Azadirachtin & B. thuringiensis & Spinosad & 76.92 & 44.44 & 60.68 a-e & 25.88 & 30.22 & 28.05 cde & 50.48 & 34.72 & $42.60 a b c$ & $43.78 \mathrm{e}$ \\
\hline 14 & Spinosad & Lambdacyhalothrin & Methomyl & 63.10 & 64.48 & 63.79 a-d & 47.05 & 23.42 & $35.23 \mathrm{bcd}$ & 35.17 & 25.79 & $30.48 c-f$ & $43.17 \mathrm{de}$ \\
\hline 15 & Indoxacarb & Spinosad & Lambdacyhalothrin & 69.23 & 48.15 & $58.69 b-f$ & 41.19 & 23.41 & $32.30 \mathrm{~cd}$ & 45.97 & 27.85 & $36.91 \mathrm{bcd}$ & $42.63 \mathrm{de}$ \\
\hline 16 & Chlorfluazuron & B. thuringiensis & Chlorpyrifos & 63.00 & 73.35 & $68.17 a b c$ & 39.52 & 34.34 & $36.93 \mathrm{bc}$ & 28.62 & 38.15 & 33.38 b-e & $46.16 \mathrm{f}$ \\
\hline$\frac{17}{18}$ & \begin{tabular}{|l|} 
Hexaflumuron \\
$B$ thuringiensis
\end{tabular} & Azadirachtin & $\begin{array}{l}\text { Deltamethrin } \\
\text { Oxymatrine +PI }\end{array}$ & $\begin{array}{l}75.38 \\
69.23 \\
\end{array}$ & \begin{tabular}{|l|}
55.60 \\
64.48 \\
\end{tabular} & $\frac{65.49 a b c}{66.85 a b c}$ & \begin{tabular}{|l|}
29.41 \\
41.19
\end{tabular} & \begin{tabular}{|l|}
29.78 \\
48.94 \\
\end{tabular} & 29.59 cde & $\frac{41.07}{39.60}$ & \begin{tabular}{|l|}
43.77 \\
1754 \\
\end{tabular} & $42.42 \mathrm{abc}$ & $\begin{array}{l}45.83 \mathrm{f} \\
46.83 \mathrm{fg}\end{array}$ \\
\hline $\begin{array}{l}10 \\
19\end{array}$ & \begin{tabular}{|l} 
D. \\
Azadirachtin
\end{tabular} & Oxymatrine + Prosuler & $\begin{array}{l}\text { Uxymadacyhalothrin } \\
\text { Lambdaser }\end{array}$ & \begin{tabular}{|l|}
09.23 \\
63.10 \\
\end{tabular} & \begin{tabular}{|l|}
04.80 \\
46.71 \\
\end{tabular} & $\begin{array}{l}0.05 \mathrm{dDC} \\
54.00 \mathrm{c}-\mathrm{f}\end{array}$ & \begin{tabular}{|l}
41.19 \\
31.18 \\
\end{tabular} & \begin{tabular}{|l|}
40.94 \\
30.22 \\
\end{tabular} & $\begin{array}{l}4500 \mathrm{dD} \\
30.70 \mathrm{cde}\end{array}$ & \begin{tabular}{|l|}
39.00 \\
50.48 \\
\end{tabular} & 34.73 & $42.61 \mathrm{abc}$ & $\begin{array}{l}40.831 \mathrm{~g} \\
42.74 \mathrm{de}\end{array}$ \\
\hline
\end{tabular}

Means followed by the same letter are not significantly different at $5 \%$ level according to DMRT test. 
The average of percent reduction of bollworms during 2008 and 2009 cotton seasons are presented in Table (6). It is clear that, the sequence number (8) repetitive spraying of spinosad in three successive sprays had the highest reduction in the number of larvae in bolls recording $48.66 \%$ reduction. While the sequence number (6) include methomyl followed by oxymatrine + prosuler and lambdacyhalothrin induced the lowest reduction recording (38.02\% reduction), the potency of these sequences could be arranged, descendingly as follows sequence number: $8>11>13>17>4>1>7>18>12>10>9>3>16>19>2>15>5>14>$ 6.

Generally, repetitive spraying of bioinsecticides in three successive sprays induced variable effectiveness against bollworms larvae, the sequence 8 (spinosad repetitive three spraying induced $48.66 \%$ reduction, sequence (B. thuringiensis) repetitive three sprays induced $41.25 \%$ reduction and sequence 10 (Azadirachtin) repetitive three sprays caused $42.55 \%$ reduction. The sequence 11 use bioinsecides, spinosad, B. thuringiensis and Azadirachtin, seq.12 use B. thuringiensis, spinosad and azadirachtin and S13 use Azadirachtin, B. thuringiensis and spinosad induced good effect in control bollworms causing $45.85 \%, 43.40 \%$ and $45.39 \%$ reduction, respectively. Using the conventional insecticides in sequence with antimolting compounds (chlorfluazuron oxymatrine + prosuler and Hexaflumuron) slightly caused high reduction in larval population which being 40.25 , 43.66 and $44.57 \%$ reduction, respectively, sequences number seq.1, seq.4 and seq.5 (Table 6). Similar observation was noticed that conventional insecticides in sequence with biocides and IGRs caused good reduction in bollworms larvae induced 39.72, 39.88, 40.19, 45.28, 43.64 and $39.94 \%$ reduction, respectively. Sequence number 14, 15, 16, 17, 18 and 19 (Table 6).

From the practical point of view, it is advisable to avoid using repetitive spraying of the same compound 3 times to minimize the selection pressure and the rate of developing resistance strain to such compound. Thus, for selecting the proper compound to be used in sequences, one should be aware of such compound have different mode of action. They belong to different chemical groups have no positive cross resistance between each other. 
Table 6. Average reduction percentages of bollworms during cotton seasons of 2008 and 2009

\begin{tabular}{|c|c|c|c|c|c|c|c|c|c|c|c|c|c|}
\hline \multirow{3}{*}{$\begin{array}{l}\text { No. of } \\
\text { seq. }\end{array}$} & \multicolumn{3}{|c|}{ No. of sequence } & \multirow{2}{*}{\multicolumn{3}{|c|}{$\begin{array}{c}1^{\text {st }} \text { spray } \\
\% \text { red after }\end{array}$}} & \multirow{2}{*}{\multicolumn{3}{|c|}{$\begin{array}{c}2^{\text {nd }} \text { spray } \\
\% \text { red after }\end{array}$}} & \multirow{2}{*}{\multicolumn{3}{|c|}{$\begin{array}{c}3^{\text {rd }} \text { spray } \\
\% \text { red after }\end{array}$}} & \multirow{3}{*}{$\begin{array}{l}\text { General mean of } \\
\text { reduction }\end{array}$} \\
\hline & \multirow{2}{*}{$1^{\mathrm{st}}$} & \multirow{2}{*}{$2^{\text {nd }}$} & \multirow{2}{*}{$3 r d$} & & & & & & & & & & \\
\hline & & & & $1 \mathrm{w}$ & $2 w$ & Mean & $1 \mathrm{w}$ & $2 w$ & Mean & $1 \mathrm{w}$ & $2 w$ & Mean & \\
\hline 1 & $\begin{array}{l}\text { Oxymatrine } \\
\text { Prosuler }\end{array}$ & + Lambdacyhalothrin & Methomyl & 59.42 & 55.83 & $57.63 \mathrm{bcd}$ & 26.10 & 29.64 & $27.87 \mathrm{~b}$ & 43.60 & 49.80 & $46.70 \mathrm{ab}$ & $43.66 \mathrm{de}$ \\
\hline 2 & Indoxacarb & Chlorpyrifos & Deltamethrin & 56.88 & 50.75 & $53.81 \mathrm{bcd}$ & 22.96 & 36.12 & $29.54 b$ & 32.60 & 41.08 & 36.84 b-e & $39.91 \mathrm{~b}$ \\
\hline 3 & Chlorfluazuron & Methomyl & Deltamethrin & 56.55 & 43.75 & $50.15 d$ & 36.42 & 49.32 & $42.87 \mathrm{~b}$ & 19.82 & 37.24 & 28.53 cde & $40.52 \mathrm{~b}$ \\
\hline 4 & Hexaflumuron & Deltamethrin & Profenofos & 65.77 & 60.83 & $63.30 \mathrm{~b}$ & 44.50 & 32.71 & $38.61 b$ & 29.82 & 33.81 & 31.81 cde & $44.57 \mathrm{efg}$ \\
\hline 5 & Chlorpyrifos & Deltamethrin & Oxymatrine + Prosuler & 55.00 & 54.75 & $54.87 \mathrm{bcd}$ & 22.21 & 31.69 & $26.95 b$ & 35.72 & 39.22 & $37.47 \mathrm{bcd}$ & $39.77 \mathrm{~b}$ \\
\hline 6 & Methomyl & Oxymatrine + Prosuler & Lambdacyhalothrin & 55.00 & 48.57 & $51.78 \mathrm{~cd}$ & 35.69 & 28.88 & $26.84 b$ & 24.80 & 35.17 & 29.98 cde & $38.02 \mathrm{a}$ \\
\hline 7 & Profenofos & Lambdacyhalothrin & Methomyl & 58.19 & 54.31 & $56.25 \mathrm{bcd}$ & 47.56 & 44.31 & $45.94 \mathrm{a}$ & 27.78 & 29.36 & 28.57 cde & 43.58 def \\
\hline 8 & Spinosad & Spinosad & Spinosad & 78.46 & 71.11 & $74.78 \mathrm{a}$ & 37.15 & 52.65 & $44.90 \mathrm{a}$ & 28.02 & 24.56 & 26.29 cde & $48.66 \mathrm{~h}$ \\
\hline 9 & B. thuringiensis & B. thuringiensis & B. thuringiensis & 56.55 & 50.00 & $53.27 \mathrm{bcd}$ & 35.24 & 32.72 & $33.98 \mathrm{~b}$ & 35.81 & 37.23 & $36.52 \mathrm{~b}-\mathrm{e}$ & $41.25 \mathrm{bc}$ \\
\hline 10 & Azadirachtin & Azadirachtin & Azadirachtin & 73.46 & 47.56 & $60.51 \mathrm{bcd}$ & 25.69 & 35.55 & $30.62 b$ & 32.11 & 40.93 & $36.52 \mathrm{~b}-\mathrm{e}$ & $42.55 \mathrm{~cd}$ \\
\hline 11 & Spinosad & B. thuringiensis & Azadirachtin & 73.46 & 53.00 & $63.33 \mathrm{bc}$ & 45.77 & 35.00 & $40.38 b$ & 34.49 & 33.06 & 33.77 b-e & $45.85 \mathrm{~g}$ \\
\hline 12 & B. thuringiensis & Spinosad & Azadirachtin & 72.26 & 50.00 & $61.13 \mathrm{bcd}$ & 40.25 & 50.89 & $45.57 \mathrm{~b}$ & 26.82 & 19.93 & 23.37 e & $43.40 \mathrm{de}$ \\
\hline 13 & Azadirachtin & B. thuringiensis & Spinosad & 68.46 & 37.22 & $52.84 \mathrm{bcd}$ & 32.61 & 32.50 & $32.53 \mathrm{~b}$ & 54.47 & 47.02 & $50.75 a$ & $45.39 \mathrm{fg}$ \\
\hline 14 & Spinosad & Lambdacyhalothrin & Methomyl & 59.05 & 55.99 & $57.52 \mathrm{bcd}$ & 43.22 & 27.56 & $35.39 \mathrm{~b}$ & 27.93 & 24.06 & 25.99 cde & $39.72 b$ \\
\hline 15 & Indoxacarb & Spinosad & Lambdacyhalothrin & 66.62 & 50.08 & $58.35 \mathrm{bcd}$ & 25.15 & 26.38 & $25.76 \mathrm{~b}$ & 45.51 & 25.54 & $35.53 \mathrm{~b}-\mathrm{e}$ & $39.88 \mathrm{~b}$ \\
\hline 16 & Chlorfluazuron & B. thuringiensis & Chlorpyrifos & 56.50 & 56.68 & $56.59 \mathrm{bcd}$ & 24.31 & 30.94 & $27.63 b$ & 26.63 & 46.08 & $36.35 \mathrm{~b}-\mathrm{e}$ & $40.19 b$ \\
\hline 17 & Hexaflumuron & Azadirachtin & Deltamethrin & 77.69 & 47.80 & $62.59 \mathrm{bc}$ & 29.35 & 41.34 & $35.34 b$ & 33.94 & 41.56 & $37.75 \mathrm{bc}$ & $45.28 \mathrm{fg}$ \\
\hline 18 & B. thuringiensis & Deltamethrin & Oxymatrine + Prosuler & 69.62 & 46.25 & 57.94 bcd & 35.24 & 41.86 & $38.55 b$ & 34.55 & 33.20 & 33.87 b-e & $43.46 \mathrm{de}$ \\
\hline 19 & Azadirachtin & Oxymatrine + Prosuler & Lambdacyhalothrin & 71.55 & 38.36 & $54.95 \mathrm{bcd}$ & 22.31 & 34.06 & $28.18 b$ & 35.58 & 37.75 & 36.66 b-e & $39.94 b$ \\
\hline
\end{tabular}

Means followed by the same letter are not significantly different at $5 \%$ level according to DMRT test. 
These results agreed with the previous finding of Johnson et el. (2000), Haidar et. al. (2002), Omar et. al. (2005), Ghure et. al. (2008) and Gosalwad et. al. (2009) showed that the newer insecticides molecules i.e. (indoxacarb, spinosad, Emamectin benzoate, lambdacyhalothrin, polytrin c and endosulfan) significantly reduced bollworms infestation in cotton. However, Emamectin benzoate was the most effective followed by spinosad.

Abd El-Mageed et. al. (2007) reported that the sequence of betacyfluthrin, malathion and spinosad and the sequence of lufenuron, malathion and spinosad induced the highest reduction (81.04 and $86.08 \%$ ) in infestation of pink bollworm larvae, respectively. Abdel-Rahman et. al. (2005) reported that the Azadirachtin Azal formulations applying at higher dose T/S (20 and $25 \mathrm{ppm}$ ) were the most effective against P. gossypiella also, Sarode et. al. (2000) and Gupta (2001) who found that Azadirachtin products and formulation of $B$. thuringiensis were effective against the bollworms and can be used as alternative to chemical insecticides. In addition, Mirmoayedi et. al. (2010) showed that three bioinsecticides, B. thuringiensis, Azadirachtin and spinosad were effective against spiny bollworm. Also, spinosad gave most effective followed by Azadirachtin and B. thuringiensis. Fadare and Amusa (2003) reported that application of microbial (biotrop, diple and thuricide) followed by chemical insecticides were better to control bollworms than other combinations evaluated. El-Metwally et. al. (2003) showed that synthetic pyrethroids (Fenpropathrin, esfenvalerate and lambdacyhalothrin) gave the highest reduction of bollworms infestation followed by IGRs, flufenoxuron, hexaflumzuron mixed with O.P chlorpyrifos, while IGRs alone gave least reduction.

On the other hand, our results disagree with that obtained by Jeyakumar and Gupta (2002) who reported Azadirachtin and Bacillus thuringiensis treatments alone was not effective against bollworms. But, treatments by neem $+B$. thurienginsis alternatively with synthetic pesticides were more effective in reducing bollworm infestation.

Histopathological effects of some biocides on pink bollworm larvae (PBW)

The histopathological structure of midgut in larvae of lepidoptera is well documented (Chapman, 1988). Figures (1 and 2) exhibit the various organs and tissues of fourth abdomen segment of the untreated 2 nd and 4th instar larvae of PBW. 


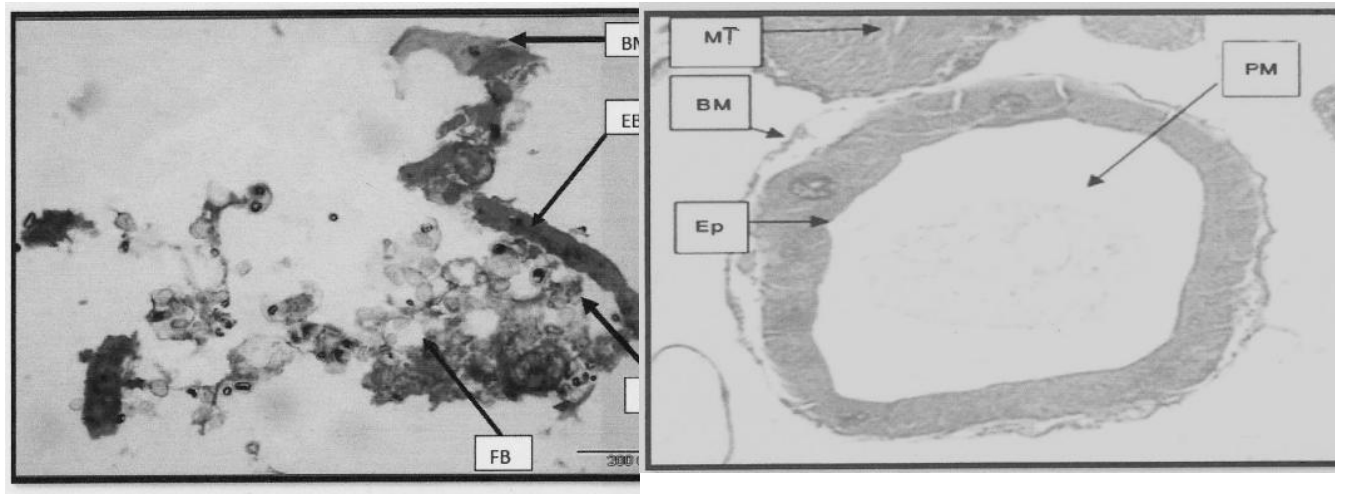

Fig. 1. Tissue and cells organs of the midgut of the 2 nd instar larvae on control (untreated)
Fig. 2. Tissues and cells organs of the midgut of the 4th instar larvae on control (untreated)

\section{The histopathological effects of $B$. thuringiensis}

Figures ( 3 and 4 ) show the effect of $B$. thuringiensis on 2nd and 4th instar larvae of PBW. These effects are complete destruction of midgut vigorous degeneration of fat bodies (IFB and OFB), sometimes degeneration of some epidermal cells and midgut in the 2nd and 4th instar larval compared with untreated larvae.

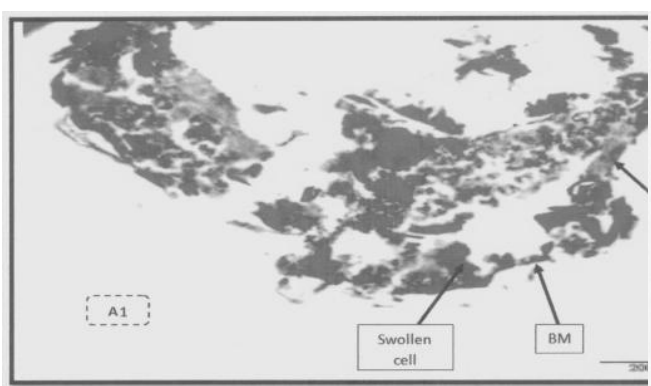

Fig. 3. The histopathological effects of B. thuringiensis $(1644 \times 102 \mathrm{IU} / \mathrm{L})$ on the tissues and cells organs of the midgut of the 2nd instar larvae

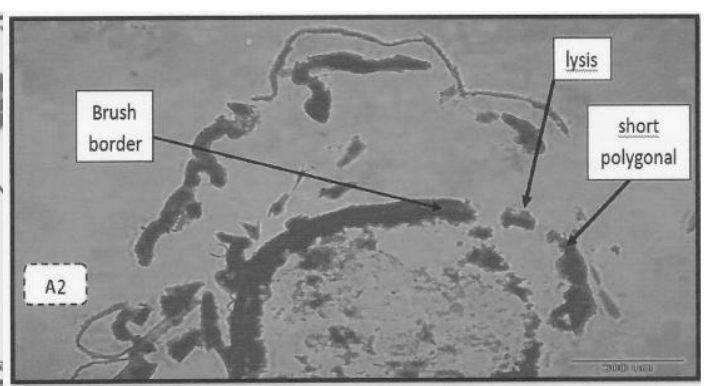

Fig. (4): The histopathological effects of B. thuringiensis $(1644 \times 102 \mathrm{IU} / \mathrm{L})$ on the tissues and cells organs of the midgut of the 4th instar larvae

\section{Histopathological effects of spinosad}

Spinosad caused adverse effects on 2nd and 4th instar larvae of PBW. Fig. (5) Shows these changes included degeneration, vacuolation and shrinkage of the epithelial cells. The lysis of the anterior mid-gut progressed through swollen clear cells vacuoles, nuclear degenerated Fig. (6) Show the serious lesion alterations included analyze and destroyed of epithelial cells and dissolve of nuclei of epithelial cells. Changes were observed in the anterior and posterior region of the midgut, included separation of the epithelial cells from the basement membrane with damage of peritrophic membrane. 


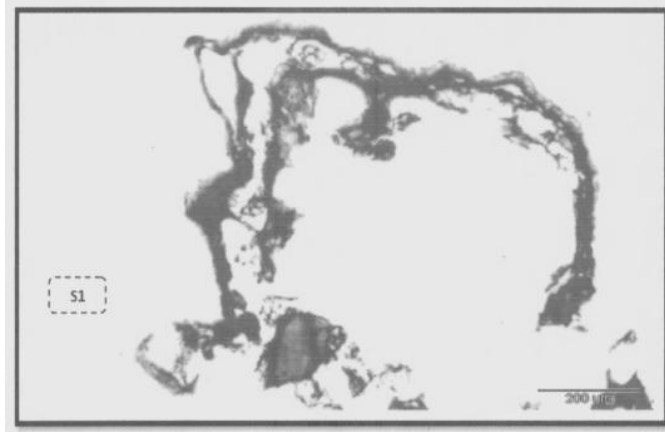

Fig. 5. The histopathological effects of Spinosad $(60 \mathrm{ppm})$ on the tissues and cells organs of the midgut of the 2 nd instar

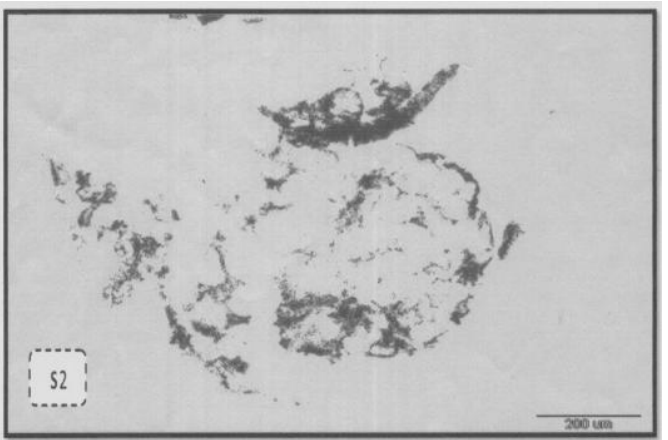

Fig. 6. The histopathological effects of Spinosad $(60 \mathrm{ppm})$ on the tissues and cells organs of the midgut of 4th instar

\section{Histopathological effects of Azadirachtin}

Figure $(7,8)$ shows the effect of Azadirachtin on 2nd and 4th instar larvae of the PBW. These effects are, figure (7) show the lysis of the anterior midgut progressed through swollen clear cells, vacuoles, nuclear degeneration, disruption of the junction of complexes and nuclear lysis, degeneration of the microvilli acceleration of the lysis of clear cells was perceptible at the level of their bruch border, basal membranes, nucleus and cytoplasmic organells, partial lysis of midgut began through local detachment among small groups of dark cells bearing a dilated basal membrane.

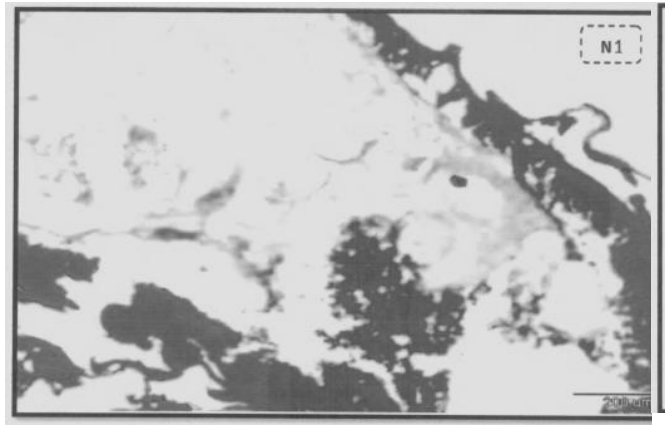

Fig. 7. The histopathological effects of Azadirachtin (4.5 ppm) on the tissues and cells organs of the midgut (2nd instar)

Figure (8) show the serious damage of the epithelial columnar cells, some cells appear slightly hypertrophied with a perceptible beginning of vacuolization at apical level cells of midgut show hardly any morphological deviation from normal ones. Generally, the histopathological effects of tested biocides were more serious on 
2nd instar larvae than 4th one Also, this previous observation was more evident when larvae were treated with field concentration of spinosad figures $(5,6)$.

The current results are in good harmony with the previous finding of Zidan et. al. (1998) who found that (MPVII) caused morphological changes to the midgut, epithelial cells layer (EP) of treated larvae of P. gossypiella and Earias insulana, Rashmi and Singh (2004) studied the histological effect of BTK on the midgut of fourth instar larvae of $\mathrm{H}$. armigera. They found that there was a hypertrophied columnar cell in the epithelium, PM pushing into sloughing cells. Hussein et. al. (2002) studied the histological and histochemical changes of vertimec and neemazalnatural product on P. gossypiella and E. insulana in laboratory conditions. They found that the two compounds caused destruction for the midgut epithelial cells. The histochemical studies revealed that both compounds affected on the polysaccharides in midgut epithelium. Also, Abdel Rahman et. al. (2005), Omar et. al. (2006) and Abd El-Halim et. al. (2008) who found that B. thuringiensis var kurstaki, Azadirachtin formulation and spinosad had histopathological effects on midgut of $P$. gossypiella and E. insulana. Abd El-Hafez et. al. (2009) found that the LC50 of B. thuriengiensis (Bt), Ecotech, Pro (BtK+Bta) and Bioclean (Beauveria basiana) had histopathological effects on midgut of PBW. Abd El-Wahed et. al. (2011) studied the histological effects of LC50 (Protecto, profect and Viruset) on midgut of 6th instar larvae of S. littoralis treated as 4th instar larvae and found that, Profect was the most effective product in causing aberrations in the midgut layers, followed by Protecto and viruset.

\section{REFERENCES}

1. Abd El-Hafez, A. Karima, A. El-Lebody, K.A. Hassan and Dina. A. Ahmed. 2009. Histopathological effect of some biocides on the fourth abdomen segment of Pectinophora gossypiella (Saund). J. Agric. Sci. Mansoura Univ., 34(5): 52595268.

2. Abd El-Hafez, Alia, A.G. Metwally, and M.R.A. Saleh. 1982. Rearing pink bollworm Pectinophora gossypiella (Saund). on kidney bean diet in Egypt. Res. Bull., Fac. Agric., Zagazig Univ., 576: 1-10.

3. Abdel-Halim, A., I.S. Abdel-Wahab and M.M. Aly. 2008. Histological effects of the plant extract Buxus chinensis (Link), Dipel 2x and Nematodes on Earias insulana (Boisd.) larvae. Egyp. J. Agric. Res., 86(2): 489-496.

4. Abd El-Mageed, A.E.M., L.R. El-Gohary and H.F. Dahi. 2007. Evaluation of several programs of sequences pesticides application on cotton bollworms and some other sucking pest in cotton field. J. Entomol., 4: 93-103. 
5. Abdel-Rahman, A.G., A.K. El-Sayed, Laila S. Hamouda and B.A.I. Imam. 2005. Histological deformations in males testes of Pectinophora gossypiella (Saund) induced by treating larvae with neem formulations. Egypt. J. Agric. Res. 83(2):

6. Abd El-Wahed, M.S., Fyza M. Ahmed, Aziza E. Abd El-Aal and Marwa, M. Abd ElAziz. 2011. The effect of certain biological agent on some biological, biochemical and histological aspects of the cotton leaf worm Spodoptera littoralis (Boisd.) Lepidoptera: Noctuidae. Egypt. J. Agric. Res., 89(2):: 431-444.

7. Burges, H.D. and N.W. Hussey. 1971. Microbial control of insects and mites Academic Press, London and New York, 861 pp.

8. Champagne, D.E., M. B. Isman and G. H. Neil towers. 1989. Inesticidal activity of phytochemicals and extracts of the -Miliaceae (In: Arnason, J.T., Philogene, B.J.R. and Morand,P.Eds, Insecticides of plant Origin .Acs Symp. Ser. 387, Washington, D.C.)

9. Chapman, R.F. 1988. The insects structure and function. 3rd edition. English Language Book Society/Edward Arnold, pp. 50-52

10. Corlett, G.R. 1961. Investigation of the effect of Bacillus thuringiensis on mammals. Canad. Wild Life Serv. Res. Proj. 1-5, Ottawa Univ.

11. Duncan, D.B. 1955. Multiple range and multiple F test. Biometrics, 11: 1-42.

12. El-Metwally, H.E., S.A. El-Mahy, Alia Abdel-Haffz and R.M. Amer. 2003. Residues of esfenvalerate and flufenoxuron in cotton bolls and the relationship between pesticide dynamics and efficacy. Bull. ent. Soc. Egypt., Econ. Ser., 29: 199-210.

13. El-Sorady, A.E.M., A.A.S. El-Zanan, M.K.A. Abo-Sholoa and A.A. El-Dahan. 1998. Influence of some insecticide sequences on natural and artificial infestation with pink bollworm Pectinophora gossypiella (Saund..). Egypt, J. Agric. Res., 76(2): 585-596.

14. Fadare, T.A. and A.N. Amusa. 2003. Comparative efficacy of microbial and chemical insecticides on four major lepidopterous pests of cotton and their insect natural enemies. African J. of Biotech., 2(11): 425-428.

15. Ghure, S.T., B.S. Kharbde and D.S. Patil. 2008. Bioefficacy of new pesticides against bollworm complex of cotton (Gossypium spp.). International J. of Plant Protection 1(2): 106-109.

16. Gosalwad, S.S., S.K. Kamble, D.W. Wadnerkar and K.B. Awaz. 2009. Efficacy of some newer insecticides for control of cotton bollworms. Journal of Cotton Research and Development, 23(2): 282-285.

17. Gupta, G.P. 2001. Role of neem in sustainable cotton pest management. National J. of Plant Improvement, 3: 37-43. 
18. Haidar, K., A. Iqtidar, A. Nazir, G.M. Aher and A. Amjad. 2002. Comparative efficacy of new vs. old chemistry insecticides for the control of Helicoverpa armigera on cotton crop. Pakistan Entomological 24(2): 121-124.

19. Henderson, C.F., and E.W. Tilton. 1955. Test with acaricides against the brown wheat mite. J.Econ. Entomol., 48:157-161.

20. Hussein, N.M., A. Gadallah and S. Tawfik. 2002. Histological and histochemical studies on the midgut of bollworms in relation to Vertimec and Neemazal. The first Conf of the Central Agrci. Pesticide Lab., 3-5, 2002, 576-588.

21. Jeyakumar, P. and G.P. Gupta. 2002. Utilization of neem and Bt for managing bollworms in cotton. Indian J. Entomol. 64(4): 424-433.

22. Johnson, D.R., G.M. Lorenz, D.J. Hopkins and M.L. Page. 2000. Comparison of new insecticides for control of Heliothine species in cotton social. Report. Arkansas Agri., Experiment Station (198): 266-270.

23. Khidr, A.A., W.M.H. Desuky, A.A. El-Sheakh and S.A. Raslan. 1996. Sequential use of some insecticides against cotton bollworms in control trials. Egypt, J. Agric. Res., 74(2): 321-331.

24. Lee, A.B. 1950. The microtonist's. VadMedcum, 11th Ed. London.

25. Menally, P. and W. Mullins. 1996. The role of prevado in western cotton IPM. Programs. Proceeding Beltwide cotton conferences. Nashville, USA, 9-12 January, pp. 859-862.

26. Mirmoayedi, A., M. Maniee and A. Yaghutipoor. 2010. Control of cotton spiny bollworm (Earias insulana). Boisd val. using three bioinsecticides Bt, Spinosad, and Neem-Azal. J. of Entomol., 7(2): 89-94.

27. Omar, R.E.M., W.M.H. Desuky, A.A. Darwish and A.E.A. Amer. 2005. Evaluation of Chinmix, Spintor and Biorepel against the cotton bollworms in the field. Ann. Agric. Sci., Moshtohor, 43(4): 1981-1989.

28. Omar, R.E.M., W.M.H. Desuky, A.A. Darwish and A.E.A. Amer. 2006. Biochemical and histological effects of Chinmix, Spintor and Biorepel compounds on larvae of pink and spiny bollworms. Ann. Agric. Sci. Moshtohor, 44(1): 279-289.

29. Rashmi, T. and N.P. Singh. 2004. Bioefficacy of commercial Btk and the histopathological changes in fourth install Helicoverpa armigera. J. Exp. Zoology, India, 7(2): 349-352.

30. Sarode, S.V., A.E. Chaudhary and V.U. Sonalkar. 2000. Evaluation of neem products against cotton bollworm complex. J. Entomol. Res. 24(4): 319-324.

31. Shaaban, A.M. and H.S. Radwan. 1974. Population dynamics of pink bollworm (Pectinophora gossypiella) (Saund) in relation to blooming and fruiting curves of cotton plants. Pf, Kranth, 4: 206-211. 
32. Temarak, S. A. 2007. Suscetibility of Spodoptera littoralis to old and new generation of Spinosyn products in five cotton. Resistant pest mangament ,vol 16, No. 2

33. Zidan, Z.H., M.I. Abdel-Megeed, Alia Abd El-Hafez, N.M. Hussein, H.M. El-Gemeiy and M.M. Shalaby. 1998. Toxicological and histological studies of Bacillus thuringiensis, MVP against larvae of pink and spiny bollworms. 7th Conf. Agric. Dev. Res., Fac. Agric., Ain Shams Univ., Cairo, Egypt, Dec. 319-332. 
فعالية تتابع بعض المبيدات على مكافحة ديدان اللوز فى القطن والتأثيرات الهستوباثولوجية لبعض المبيدات الحيوية على دودة اللوز القرنفلية

محد عبد المحسن سلامة1 ، محمد عبد السلام عبد الباقى ، جيهان بدوى أحمد النجار2 2 عصام يوسف عزب النجار 2

$$
\text { r . . كلبة الزراعة ـ جامعة كفرالشيخ }
$$

2008 ، 2009م فى مزرعة محطة البحوث الزراعية

$$
\text { أجريت هذه التجارب فى موسم }
$$

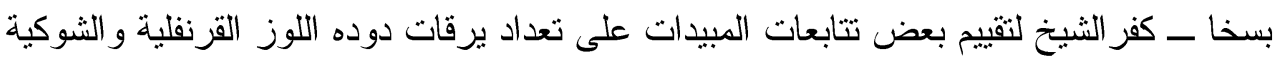
وكذلك النسبة المئوية للإصابة فى اللوز الأخضر وذلك بهدف الحصول على أحسن تتابع فى مكافحة

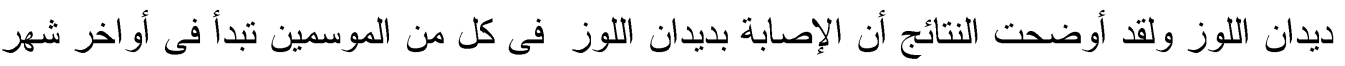

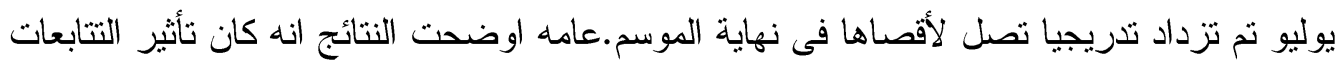

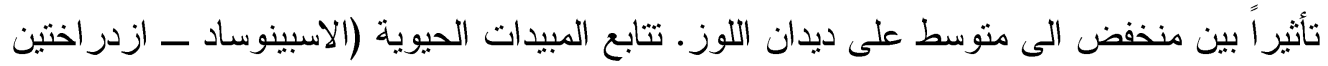

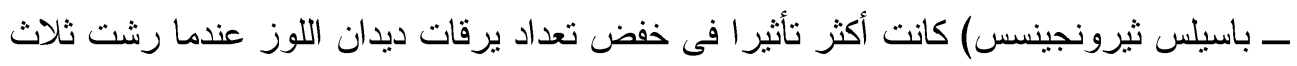

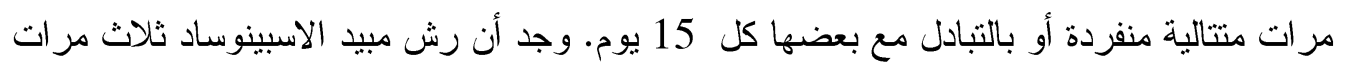

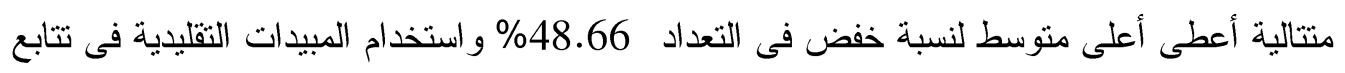

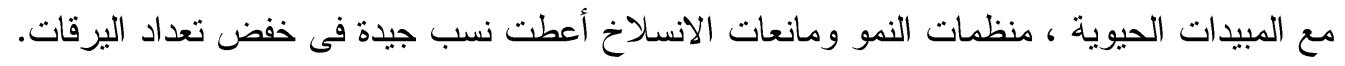

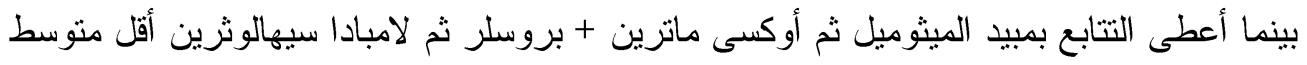

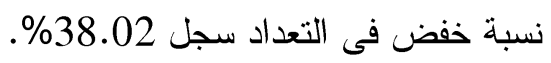

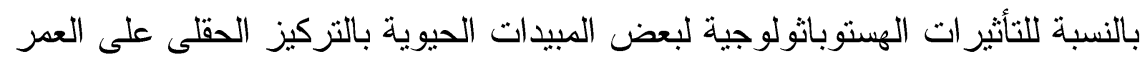

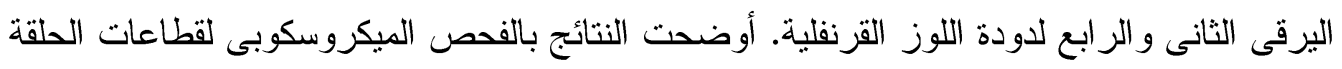

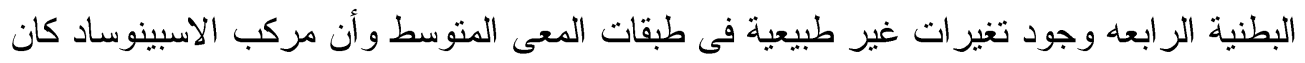

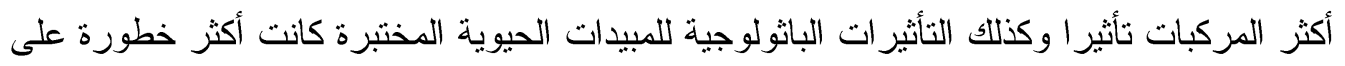

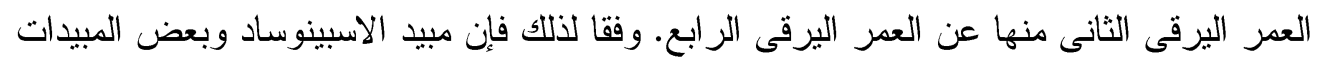

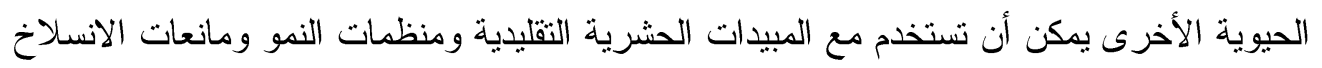

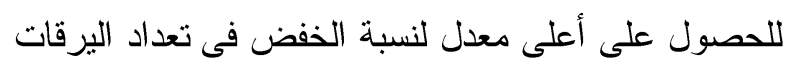

